ISSN: $1130-3743$

\title{
LA REGIONALIZACIÓN DE LA EDUCACIÓN COMO TECNOLOGÍA COGNITIVA VIRTUAL
}

\author{
The regionalization of education as a virtual \\ cognitive theory
}

\author{
Antonio J. COLOM CAÑELLAS \\ Facultad de Educación. Universidad de las islas Baleares. \\ Campus Universitario. 07071 Palma de Mallorca
}

Fecha de aceptación del original: 1997

BIBLID [(1130-3743) 9, 1997, 7-19]

RESUMEN

La influencia de las nuevas tecnologías en la educación nos obliga a una redefinición conceptual de la misma. Tras analizar los efectos de la virtualidad en el campo de la educación, de la escuela y de los sujetos, se concibe la Teoría de la Educación como una tecnología cognitiva de carácter no trivial. Sólo así se plantea una educación para la innovación del conocimiento capaz de contemplar la creatividad y con ella las atribuciones propias del pensamiento sistémico. Además se confirman estas tesis con las últimas investigaciones de la neurociencia referidas al hemisferio cerebral derecho.

\section{ABSTRACT}

The influence of new technology on education brings with it the necessity of a conceptual redefinition of the term. After analyzing the effects of virtuality in the field of education, in the school, and on the individuals, we can conceive the Theory of Education as a cognitive technology of no trivial character. Only in this way can we design an education for the innovation of knowledge acquisition capable of contemplating creativity and along with in the attributions characteristic of systemic thought. Furthermore, these theses are being confirmed by the latest findings in neuroscience referring to the right hemisphere of the brain. 
Es necesario arbitrar un nuevo espacio para la educación, o lo que es lo mismo, regionalizar la educación en su adecuada ubicación, vistos los cambios que en la sociedad y en todos los órdenes del conocimiento se avecinan. Pestalozzi, Herbart, y en general la pedagogía de la modernidad, fiel a la mentalidad burguesa-liberal y a la moral protestante — convenientemente adaptada al catolicismo en los países latinos-generaron una razón pedagógica que desde la racionalidad filosófica o desde la racionalidad experimental intentó adecuarse a las necesidades sociales, a las innovaciones científicas y al sentido moral de la Bildung o de las axiologías dominantes. Sin embargo, otros modelos sociales, nuevos paradigmas científicos, o el sentido de formación que hoy se demanda, solicitan otros planteamientos en el concepto y en la racionalidad educativa. Y en este nuevo contexto, las tecnologías de la información, como ejemplo de uno de los cambios importantes acaecidos en estos últimos años, juegan y están llamadas a jugar un nuevo y determinante papel.

Evidenciaremos lo mencionado con un cotidiano ejemplo. De ser la tecnología considerada estructurante de la sociedad de masas y de la domesticación capitalista tal como se afirmaba en los años sesenta ${ }^{1}$, se nos muestra cara al futuro con un papel diametralmente opuesto al considerarse estímulo para el desarrollo de las capacidades individuales, hasta tal punto que sólo es el discurso de la modernidad el que acusa a la tecnología de alienadora 2 . El ejemplo de la TV y el video, creo que diluyen cualquier duda en este sentido; la TV es masiva - todos ven lo mismo en un momento determinado- en cambio, el video, personifica, cada uno ve lo que quiere y cuando quiere, y en este sentido, anula o reduce el poder masivo de la TV. En este sentido, la perspectiva de la TV a la carta es también para-

1. De alguna manera, gran parte de la bibliografía típica de la década de los años sesenta sobre medios de comunicación de masas se hace eco de la crítica a los mass media. También y sobre todo en Europa, esta crítica adquiere connotaciones ideológicas importantes tal como sucede en dos de los autores más representativos de esta corriente a la cual me refiero. A pesar de los años transcurridos véase como ejemplo de lo que decimos las obras de ENZENSBERGER, H. M.: Elementos para una teoria de la comunicación. Anagrama, Madrid 1972, y Habermas, J.: Ciencia y técnica como ideología. Edit. Tecnos, Madrid, 1984.

2. Es el tema ya clásico de los apocalípticos y de los integrados, siguiendo con la terminología de U. Eco; en estos momentos sería Habermas el autor paradigmático defensor de la posibilidad humanística y de los valores sociales, en contra de las alineaciones tecnológicas, al considerar que la Ilustración no está aún concluida, lo cual, desde una perspectiva puramente historicista, no deja de ser como mínimo una afirmación realmente curiosa. Véase, como sustento de la tesis de Habermas su obra El discurso filosófico de la modernidad. Edit. Taurus, Madrid, 1991. Para un conocimiento más profundo y completo de su pensamiento recomendamos Teoría de la acción comunicativa (2 vols.). Edit. Taurus, Madrid, 1988. En cambio, la opinión que concibe la tecnología como favorecedora de las posibilidades del hombre se encuentra en toda la literatura de la postmodernidad; véase a modo de ejemplo: Finkielkraut: La derrota del pensamiento. Anagrama, Barcelona, 1987; Foster, HABERMAS, BAUdRILlARD \& OTROS: La postmodernidad. Edit. Kairos, Barcelona, 1985; JAMESON: El postmodernismo o la lógica cultural del capitalismo avanzado. Edit. Paidós, Barcelona, 1991; KLAPPENBACH, A.: Ética y postmodernidad. ICE de la Univesidad de Alcalá de Henares, Madrid, 1990; Kung: Proyecto de una ética mundial. Edic. Trotta, Madrid, 1991; LyoTARD, J. F.: La condición postmoderna. Edit. Cátedra, Madrid, 1987; VATtimo: El fin de la modernidad. Edit. Gedisa, Barcelona, 1986 y también de este mismo autor: La sociedad transparente. Edit. Paidós, Barcelona, 1990. 
digmático del cambio que las nuevas tecnologías procuran de los usos de la propia tecnología. Las nuevas tecnologías, se intuyen ahora, a disposición del hombre para desarrollar y profundizar todos los ámbitos e indagar y culminar sus capacidades expresivas, creativas e intelectuales.

Asimismo, la educación de la modernidad, al igual que todas las pedagogías críticas y liberadoras, han tratado de la libertad humana como un aspecto inviolable de la acción pedagógica, si bien al mismo tiempo, han ido convirtiendo sistemas educativos triviales, predefinidos funcionalmente. La superación de esta contradicción estriba no tanto en la continuidad de los discursos liberadores cuanto en la capacidad de la educación del logro de la no trivialidad, o sea, de asumir la libertad psíquica y mental del sujeto3. La verdadera libertad no es ideológica, es cognitiva.

\section{LA VIRTUALIDAD PEDAGÓGICA}

Acaso, el cambio al que nos referimos, se inició con la crisis del petróleo a principio de los años setenta a través del papel que desde entonces fue jugando la OPEP (Organización de países exportadores de petróleo), al costreñir la industria y la economía occidental y darse cuenta, ésta, de su casi plena dependencia energética. Este hecho, muy posiblemente, fue quien produjo la inflexión en el cambio tecnológico, acelerando la denominada "lógica de la necesidad" que tuvo su respuesta en las nuevas tecnologías, que se distinguen, fundamentalmente, porque se basan en la información y en el conocimiento, sin generar apenas gasto energético ni consumo de materias primas de alto valor económicót.

Sin embargo, además de los efectos económicos y de las nuevas estrategias industriales, hay, a nivel antropológico, consecuencias e incidencias de gran interés que las ciencias de la educación no pueden obviar por más tiempo; me refiero al hecho de que la tecnología va transformando también nuestras mentes porque de alguna forma accedemos a los datos y a las imágenes mentales de diferente manera; paralelamente, cambiamos nuestro modelo mental de la realidad y nuestra representación del mundo, ya que al llegarnos más información y más rápidamente, nos vemos en la tesitura de ir cambiando nuestras imágenes más anticuadas, por lo que nuestras consistencias son cada vez más transitorias e instantáneas.

3. La cuestión de la educación en relación a los sistemas triviales y no triviales, así como el estudio de las características de los mismos ha sido acaso una de las contribuciones más concretas, y por ello más importantes, que ha realizado N. Luhmann a la cuestión educativa. Véase por ejemplo de este autor: "Presupuestos estructurales de una pedagogía reformista. Análisis sociológico de la pedagogía moderna", en Revista de Educación $\mathrm{n}^{\mathrm{Q}}$ 291, Madrid, enero-abril 1990; LuHMANN, N. \& SCHORKI, K. E.: El sistema educativo. Problemas de reflexión. Instituto Tecnológico y de Estudios Superiores de Occidente, Universidad de Guadalajara y Universidad Iberoamericana, México, 1993; LUHMANN, N.: Teoria de la sociedad y pedagogía. Edit. Paidós, Barcelona, 1996.

4. He tratado estas cuestiones últimamente en: Colom, A. J. \& Melich, J. C.: Después de la Modernidad. Edit. Paidós, Barcelona, 1995; véase asimismo: Colom, A. J. \& Melich, J. C.: «Postmodernidad y educación. La teoría de Toffler y la práctica de la CMU», en Teoría de la Educación, nº 5, Salamanca, 1993. 
Además, las nuevas tecnologías individualizan y desmasifican, lo que conduce a la desuniformización de nuestras mentes refrendando entonces el desarrollo de la creatividad, y en definitiva, la génesis de nuevas informaciones y conocimientos. La industria espacial, la inteligencia artificial, la robótica, la ingeniería genética, la biotecnología... etc., son sin duda algunos de los horizontes en los que la predicción efectuada es en parte realidad.

El conocimiento, entonces, se evidencia como el gran motor del futuro, por lo que la estructura que da origen y expande el conocimiento - la educación-demanda la concreción de una nueva racionalidad pedagógica. La educación, y con ella el conocimiento, se convierten en la carga de alimentación, en la verdadera energía de la tecnología, necesaria para su puesta en marcha y posterior desarrollo.

Las tecnologías de la información no tienen valor en su física, en tanto que conjunto de artefactos que pueden suponer, más o menos, una novedad material en el aula, y ser por tanto y en cierta forma, un elemento estimulador, motivador o facilitador; la tecnología de la información aporta, como verdadera novedad, la capacidad de transmitir de forma diferente un cuerpo de conocimientos, al mismo tiempo que coayuda a formar un pensamiento caracterizado fundamentalmente por el desarrollo de procesos 5 .

Esta característica, asentada en la capacidad cognitiva procesual, presupone hablar de la información, no sólo en su vertiente acumulativa, sino en una perspectiva más estructurante. La información, mediante el uso de las nuevas tecnologías, se reconvierte en un instrumento de organización de los contenidos, por lo que se nos presenta, cognitivamente, activa y funcional. Con ello, llegamos a uno de los puntos básicos y definidores de la nueva funcionalidad de los sistemas educativos; nos referimos al hecho de que la educación tendrá una finalidad muy concreta y es que, en este contexto tecnológico y gracias a él, deberá facilitar antes que otra cosa, no sólo el conocimiento, sino la posibilidad de transformar los conocimientos, por lo que, conocer, será innovar. En este contexto, no hay duda que la utilización continuada del ordenador es una forma inédita de desarrollar y estimular nuestras capacidades intelectuales ya que, de hecho, amplía y multiplica las posibilidades de nuestro cerebro al controlar múltiples variables en períodos muy cortos de tiempo.

Todo ello hará que se produzca un cambio en el seno de la teoría educativa que conllevará a su transformación, incluso conceptual y epistemológica. Las ciencias de la educación, en el futuro, deberán indagar y estudiar los sistemas de comunicación de información que evidencien capacidad de restructuración cognitiva y de modificación de nuestros procesos mentales; se tendrán que basar en sistemas propiciadores de información con potencial cognitivo. Y es que las nuevas tecnologías se reconvierten en simuladores cognitivos, que tendrán que ser objeto

5. VÁzQUEz, G.: "Inteligencia, tecnología y escuela en la sociedad post-industrial", pp. 207 a 252 de: McCinNTock, R., STREIBel, M. \& VÁzQuez, G.: Comunicación, tecnología y diseños de instrucción. La construcción del conocimiento escolar y el uso de los ordenadores. CIDE, Ministerio de Educación y Ciencia, Madrid, 1993, p. 239. 
de estudio de la futura Teoría de la Educación. Desde esta perspectiva, la educación y con ella su estructura teórico-epistemológica, se nos presenta como una verdadera tecnología cognitiva de carácter no trivial, o sea, no sólo en sentido instructivo - de aprendizaje de contenidos - sino también en sus dimensiones más ontológicas, máxime cuando la adquisición de hábitos, de valores, o de creencias y actitudes está afectada por las capacidades cognitivas de los sujetos. Y tal afirmación no debe hacernos rasgar las vestiduras ya que la tecnología, como afirma G. Vázquez ${ }^{6}$, "puede $y$ debe entenderse como una cierta forma de pensar característicamente bumana. La pedagogía se nos presenta pues como una ciencia del hacer cognitivo no trivial, en base al estudio de los fenómenos y sistemas capaces de propiciar - mediante la educación- la potenciación cognitiva del hombre en un contexto de libertad decisional. Y es que hablar de una tecnología de la cognición implica abogar definitivamente por la concepción no trivial del sistema educativo, es decir, por su indefinición funcional y por abrir de par en par las posibilidades del ser humano. El concepto de libertad que es inmanente a la no trivialidad sistémica está ahora en la nueva epistemología pedagógica asegurado por la capacidad abierta e indefinida de la cognición humana.

La educación, bajo el contexto diseñado anteriormente, deberá, de forma incuestionable, transformarse y cambiar, a fin de adaptarse a las nuevas necesidades y condiciones de la sociedad próximamente inmediata. Ello implica afirmar que los objetivos de la educación no podrán ser ya los actuales, surgiendo como finalidad esencial de los mismos, el aumento de la capacidad de adaptación del individuo al cambio continuado. La sociedad está precisando, de cada vez más, personas capaces de adaptarse a la multiplicidad de situaciones que la actual vida contemporánea va diseñando, así como a la constante innovación tecnológica que poco a poco va al mismo tiempo transformando mores, hábitos y costumbres; el hombre educado deberá ser aquella persona capaz de controlar estos cambios al mismo tiempo que implementar otros nuevos para así dar soluciones a problemas inéditos emergentes; en todo caso, el hombre educado, deberá saber vivir en una sociedad en constante devenir que tanto la investigación como el conocimiento irán propiciando continuamente. Ello supone afirmar que una educación para el cambio necesita profundizar en las posibilidades cognitivas del sujeto ya que las soluciones adaptativas se encuentran en los recursos y capacidades mentales e intelectuales. De ahí que veamos el estudio de la educación centrado cada vez más en las tecnologías cognitivas, o sea en los medios y sistemas capaces de desarrollar las capacidades cognitivas del hombre, o si se quiere, la no trivialidad.

Para ello y como afirma R. McClintock ${ }^{7}$, será además necesario reorganizar la cultura para adaptarla al uso de las tecnologías digitales, lo que significará un cambio en los planteamientos curriculares que deberán bascular ahora sobre una

6. Ibidem, p. 232.

7. McClrntock, R.: "Elaboración de un nuevo sistema educativo", pp. 127 a 176 de: McCunTock, R., STReibel, M. \& VÁzQuez, G.: Comunicación, tecnología y diseños de instrucción. La construcción del conocimiento escolar y el uso de los ordenadores. CIDE, Ministerio de Educación y Ciencia, Madrid, 1993. Véase en concreto, p. 146. 
nueva concepción del saber. Deberá plantearse una cultura educativa que mire fundamentalmente al porvenir. La educación no debe ser ya la transmisora de la cultura del pasado y la pervividora de la historia en las nuevas generaciones. Tampoco debe ser comprensiva del presente ya que éste no es perdurable; el presente, el momento, debe servir como mecanismo didáctico para explicar el cambio y conseguir así el objetivo prioritario de la educación: la movilidad, la adaptación a lo nuevo, a lo cambiante, por lo que la educación se debe plantear, como el desarrollo del potencial cognitivo cara a las previsiones del futuro. La sociedad del aprendizaje no es nada sino conduce a la sociedad del saber y del conocimiento: "los gobernantes de mañana tendrán que inventar y sobre todo deberán permitir inventam 8 .

Será necesario, en consecuencia, crear un nuevo sistema educativo y dotar a las escuelas de nuevas funciones que deberán contemplar el plano instructivo entendido ahora como creación de conocimiento. Tecnológicamente, el cambio estribaría en superar nuestra escuela actual, asentada en el feed-back, o sea, en la capacidad de adaptación a la realidad profesional, social, axiológica... etc., para lograr una escuela que concibiese la educación como la inclusión en los educandos de la capacidad de feed-before; o sea, del control proyectivo. Yo creo que este es el gran reto ante el cual debemos prepararnos y el gran cambio que debemos posibilitar en nuestras instituciones y, sobre todo, en el hombre.

Plantear un sistema educativo funcionalmente orientado al logro del feedbefore educativo es instaurar un sistema que inculque el control de las variables hipotéticas que en un momento dado puedan darse; es, en suma, inculcar la capacidad de intuir el futuro y estar entonces preparado para su advenimiento; el feedbefore, supone, mediante la información multivariada que se posee, dotar al alumno, al hombre, de capacidad anticipatoria, de tal manera que la utilización de la información estribe en proyectar acciones y conductas a fin de enfrentarse con éxito y eficacia a situaciones que se darán en el futuro.

Si el sistema educativo de la modernidad se asentaba en la dotación de feedback, o sea, de adaptación, de integración a un medio, con la ayuda de las nuevas tecnologías de la información, los sistemas educativos que seguirán a la modernidad, deberán aportar capacidad de feed-before, o si se quiere, de conocimiento del futuro, de control proyectivo o de capacidad anticipatoria9. Para ello,

8. Proféticas palabras de A. Danzin, en una obra ya clásica pero que no merece en absoluto el olvido; me refiero a Science et renaissance de l'Europe, Paris, Edit. Chotard, 1976.

9. Es difícil encontrar ejemplos de conductas propias del feed-before; normalmente, siempre se plantea el símil del portero ante un lanzamiento de un penalty o falta máxima; teóricamente, por la velocidad que lleva el balón y por los niveles de visión del ojo humano, el penalty, si está bien lanzado, es imparable; sin embargo, en muchas ocasiones, gracias a la información que posee el portero de la forma y manera como el delantero suele tirar las faltas máximas, el portero puede intuir o predecir la trayectoria futura del balón y así controlar el sentido de su dirección. Fíjese el lector que el posibilismo del control anticipatorio o feed before, depende en exclusividad de la información o el conocimiento previo que de una situación o fenómeno se posee; como se ve, una vez más, la información sirve para controlar o conocer situaciones, lo que ocurre es que en este caso las situaciones y la información no se dan al unísono, en el momento, como en los casos de ajuste o feed-back, sino que aquí, se posee la información previamente de acontecimientos que se vislumbran en el futuro, por 
es necesaria la utilización multivariada de información y la experiencia en situaciones y en toma de decisiones, o sea, implica transformar las aulas en verdaderos laboratorios de simulación; así, los medios informáticos, las técnicas de juegos, las previsiones del azar, la prospectiva... etc., se nos presentan como los pilares en donde fundamentar la nueva pedagogía en tanto que tecnología cognitiva.

\section{LA VIRTUALIDAD ESCOLAR}

Hay otra característica que no podemos dejar de lado y que afecta a la educación y a la escuela en su conjunto, y es que en la "Sociedad del conocimiento" a la que nos abocamos, se dará definitivamente la pérdida, por parte de los sistemas educativos, del monopolio del saber y de la formación; las tecnologías informativas han democratizado el conocimiento y han roto las barreras de los espacios informativos y formativos. Hoy, la sociedad, toda ella, es espacio de conocimiento, por lo que forzosamente, la escuela ha dejado de ser la institución que aún gozaba de tal privilegio; ello hace que debamos reflexionar qué papel puede y debe jugar la escuela ante la nueva situación planteada.

"Veo una nueva funcionalidad en la institución escolar, o si se quiere, una misión que cumplimentar por ahora inédita, y que yo centraría en convertirla en un espacio de síntesis, o si se quiere, en un espacio de espacios. Con ello quiero significar a la escuela, y lo introduzco a modo de símil, con la misma definición que en sus Principia... daba B. Russell a la noción de número a la hora de concebirlo como la clase de clases $^{10}$.

¿Qué significa entender la escuela como espacio de síntesis o como espacio de espacios? En una sociedad culturalizada, por múltiples medios y en múltiples espacios - la empresa, los media, la ciudad ${ }^{11}$, los centros culturales, los museos... etc.- la escuela debe ser el espacio abarcador de todos los demás espacios educativos a fin de posibilitar una síntesis de todos los mensajes recibidos por otras vías. Síntesis en cuanto a reestructuración de mensajes y en cuanto a re-ordenación de las informaciones recibidas, síntesis que dará sentido a la cultura en mosaico que vamos recibiendo. La escuela deberá fundamentalmente aportar una lógica de la cultura en el niño.

lo que se puede hablar de control anticipatorio o proyectivo. De alguna manera, el funcionamiento de los misiles es similar; de ahí su eficacia, ya que poseen fuentes de información anticipada sobre los "comportamientos" de sus objetivos, por lo que proyectan con seguridad su trayectoria.

10. En sus Principia Matemática B. Russel definía el número 1 como la clase abarcadora de todos los elementos únicos o unidades, el número 2 como la clase abarcadora de todos los elementos duales, el número 3 como la clase abarcadora de todas las tríadas, el número 4 como la clase abarcadora de todos los cuartetos, y así sucesivamente. No hay duda entonces que desde esta perspectiva el "número" es la clase que abarca a todas las clases de unidades, de dualidades, de tríadas, de cuartetos, de quintetos... etc., o sea, "la clase de clases".

11. Sobre el posibilismo de la ciudad educativa véase el texto publicado por el Ayuntamiento de Barcelona, en ocasión del Primer Congreso Mundial de Ciudades Educadoras: La ciudad educadora. Ayuntamiento de Barcelona, 1990. Asimismo el autor ha tratado tal cuestión en algunas ocasiones; sirva a modo de ejemplo: Colom, A. J.: "Educación y Municipios", pp. 33 a 54 de: Castillejo, J. L., Colom, A. J. \& ALTER: Condicionamientos socio-políticos de la educación. Edit. Ceac, Barcelona, 1985. 
De esta forma, concebir la escuela como espacio de síntesis de múltiples espacios culturales y de información es refrendar su capacidad estructurante y constructivista, pues deberá ser espacio creador de significados antes que espacio transmisor de informaciones. A la escuela se podrá llegar con información y en la escuela se tendrá que analizar, indagar, trabajar con esta información para reordenarla y sintetizarla, para en definitiva, estructurarla dándole un sentido personal, propio e individual, que sirva para iniciar la aventura de búsqueda de nuevos conocimientos. Éste será, fundamentalmente, el objetivo del nuevo ámbito escolar, que por ello mismo, se convertirá, tal como decíamos, en espacio de espacios, al tener que simular e integrar los aportes culturales recibidos en otros lugares, especializados o difusos, de recepción de información. O sea, a una pedagogía entendida como tecnología cognitiva le corresponde una escuela en tanto que espacio para el desarrollo de la cognición. Para ello proponemos tres estrategias básicas de cambio:

- Cambio de la estructura docente actual. Si la escuela es espacio de síntesis, tendrá que desarrollar a su vez síntesis de las fuentes informativas; el profesor pierde su monopolio, y cualquier ciudadano, al igual que cualquier media, puede ser fuente de formación e información y por tanto aprovechable para los intereses escolares.

- Revolución en los contenidos o programas, ya que de cada vez la sociedad marcará más concretamente sus necesidades; en este sentido la escuela será síntesis entre formación e información, sirviendo de puente entre las necesidades sociales y las individuales.

- Enfoque del conocimiento hacia el futuro, o escuela del feed-before.

La consecución de estos tres hitos daría como resultado el logro de la nueva educación y de la nueva condición escolar. Cualquier otra innovación educativa que no incidiese radicalmente en línea de estas transformaciones, serían soluciones planteadas en el seno del sistema escolar de la modernidad, lo que, en todo caso, supondría mejorar la educación para logros u objetivos que nada tienen que ver con el futuro ni con el objeto de la adaptación al cambio que se exige ya a partir de nuestro actual tiempo y momento. El futuro se asienta en el conocimiento, en el saber, que será sin lugar a dudas la nueva mercancía del futuro. El producir se fundamentará más que nunca en el conocimiento y conocer significa por otra parte innovar. En este contexto, una escuela y una educación para el cambio implica como única solución poseer una educación y una escuela que patrocinen ellas mismas la posibilidad de la innovación, y ésto sólo puede conseguirse mediante el desarrollo cognitivo.

El conocimiento se nos presenta entonces como el vector determinante del mundo económico, y en consecuencia, de la realidad en todos sus órdenes. Vamos desembocando a la necesidad permanente de formación y nos orientamos hacia un mundo en donde conocer el conocimiento (o lo que nosotros llamamos conocimiento virtual) será el valor indispensable para la supervivencia. La necesidad del conocimiento se entronca a su vez y como vamos viendo con el desarrollo de una sociedad asentada cada vez más en la tecnología y en sus efectos. 


\section{LA VIRTUALIDAD COGNITIVA}

Tal como hemos visto, el conocimiento, o mejor dicho, la innovación del conocimiento, será el componente principal del desarrollo de la sociedad en todos sus órdenes ${ }^{12}$. Como dice S. Giner, la innovación ha dejado de estar en manos de la técnica para ser fruto del cognitivismo sistemático ${ }^{13}$. Ello implica, qué duda cabe, revolucionar el conocimiento.

Innovar el conocimiento es crear nuevo conocimiento, y ésto, la creación de nuevo conocimiento, no puede darse por medios que no sean "nuevos". Los nuevos conocimientos serán realmente nuevos si afectan a contextos asimismo novedosos, por lo que la innovación sólo puede llegar a través de entornos innovadores. Consecuentemente, y aquí está la gran aportación reflexiva, la innovación no puede llegar de la "realidad natural"; en efecto, la ciencia en la modernidad, se ha desarrollado en tanto que discurso o lenguaje acerca de la naturaleza y de los fenómenos o procesos concurrentes en ella. Ello significa, que tomar a la naturaleza como el objetivo del conocimiento, no supone la creación de conocimiento innovador, ya que lo que realmente se conseguiría, siguiendo investigando las relaciones y las fenomenologías naturales sería ampliar el conocimiento tradicional, un conocimiento que si bien sería "novedoso" o "nuevo" - no conocido- no obstante no sería innovador. Sería, en definitiva, una ampliación de un tipo de conocimiento científico propio de la modernidad y que se ha venido en denominar "conocimiento natural".

O sea, que para crear no sólo nuevo conocimiento sino conocimiento realmente innovador - otro tipo de conocimiento o conocimiento virtual- debemos aplicarnos al estudio de otras realidades, o si se quiere, de realidades no naturales. Ello implica fundamentar la innovación en el estudio de otros objetos nuevos, creados ad hoc, o sea, artificiales. En este sentido, pues, sólo la realidad artificial puede plantearse como el contexto creador del conocimiento innovador.

Ahora bien, tal como hemos visto, la creación de nuevo conocimiento, lo que podríamos denominar conocimiento virtual, depende, forzosamente, de la creación de nuevos entornos o ambientes de conocimiento ${ }^{14}$. El salto está, evidentemente, en superar el conocimiento de la naturaleza, o de lo natural, para lograr "otro tipo de conocimiento"; téngase en cuenta que la investigación de la naturaleza alumbró la ciencia, con sus leyes y su capacidad descriptiva de los fenómenos; en cambio, la investigación de efectos artificiales ha traído consigo la tecnología, o invención de nuevos artefactos que propician a su vez nuevas aplicaciones y nuevas formas de resolver problemas o situaciones, creando entonces y en consecuencia nuevos entornos, o al menos, modificándolos en parte. De ahí que, hoy en día, la innovación esté en el diseño y a su vez se diga que el diseño es de por si innovador. No nos extrañe entonces que la primera condición de la innovación sea, en opo-

12. Servan-Schreiber, J. J. \& Crecine, B.: La revolución del conocimiento. Edit. Plaza y Janés, Barcelona, 1986, p. 41.

13. Véase Giner, S.: Ensayos civiles. Edit. 62, Barcelona, 1987, p. 41.

14. En este sentido véase: Colom, A. J.: "Tecnología, educación y conocimiento virtual”, pp. 17 a 25 de Tecnología y comunicación educativa, nº 22, México, enero-marzo, 1994. 
sición a la realidad natural o dada, crear realidades artificiales o si se quiere "no naturales" a fin de que cumplan el papel de ser estructuras contextualizadoras, estimuladoras e instrumentalizadoras del nuevo conocimiento. El futuro, de ello no hay duda, se encuentra en el desarrollo de lo que ya hace años, H. Simon, denominara la "ciencia artificial", 15 .

Aquí, precisamente, es donde se encuentra el gran papel a desarrollar por las tecnologías de la información ya que es precisamente, gracias a las nuevas tecnologías, que se forman nuevos ambientes artificiales y donde en consecuencia surge la posibilidad del conocimiento innovador, o si se quiere, del conocimiento virtual. En este sentido, el conocimiento innovador se nos presenta como el objeto prioritario de la pedagogía entendida como tecnología para el desarrollo cognitivo.

Un centro educativo computerizado presupone la creación de un espacio pedagógicamente artificial, ya que las interacciones propias de la adquisición del conocimiento se desarrollan por y mediante la asistencia del ordenador; ello, evidentemente, además de acercarnos a la tecnocultura, nos propicia una forma de aprendizaje, mediante un artefacto artificial que, por ello mismo, conlleva la posibilidad de conocimiento y del cognitivismo virtual.

La conclusión es clara: el uso de las nuevas tecnologías en los aprendizajes implica la generación de nuevas perspectivas cognitivas, ya que la utilización, por ejemplo del ordenador, implica generar inteligencia artificial al desarrollar esfuerzo y funcionalidades mentales sobre un mecanismo no natural. La utilización del ordenador abre al hombre nuevas perspectivas intelectuales y actualiza otras capacidades cognitivas hasta ahora no desarrolladas; es obvio que ante nuevas situaciones el hombre ensaye nuevas soluciones, con lo que el ordenador y la nueva situación de interacción que supone su manejo, obliga a producir nuevas estrategias cognitivas y a la larga, nuevas perspectivas de utilización de los recursos mentales. O sea, una enseñanza asistida por ordenador, al crear un nuevo entorno (y será un nuevo entorno por ser un entorno artificial), crea nuevas perspectivas cognitivas, y por tanto, se plantea como conocimiento virtual, o si se quiere, conocimiento con potencialidad y capacidad innovadora.

El aprendizaje instrumentalizado tecnológicamente implica reconvertir esta tecnología en un medio facilitador de nuevas perspectivas cognitivas por lo que la educación tiende a convertirse, tal como enuncíabamos, en una tecnología cognitivista y por tanto en una tecnología propia de la construcción humana.

Posibilismo éste que viene además refrendado por las últimas aportaciones neurofisiológicas; en efecto, poco a poco vamos conociendo las capacidades mentales del hemisferio cerebral derecho, que por cierto se oponen al desarrollo intelectual que la escuela actual está propiciando, centrada, fundamentalmente, en el desarrollo de los posibilismos mentales propios del hemisferio izquierdo, o sea, el lenguaje, las secuenciaciones lineales, la capacidad de análisis y la discriminación. Sin embargo, el hombre posee otras capacidades, que la escuela margina de sus intereses, tales como las imágenes, las pautas, la capacidad de síntesis, las visio-

15. Simon, H.: Las ciencias de lo artificial. Edit. ATE, Barcelona, 1973. 
nes globales o de conjunto, la simultaneidad, la conectabilidad... etc., que se aproximan a lo que bien podríamos denominar "pensamiento sistémico" 16 y que son fruto de las capacidades controladas por el hemisferio cerebral derecho ${ }^{17}$. Pues bien, la utilización del ordenador, conlleva toda una serie de estrategias cognitivas, que vienen dadas por las aportaciones novedosas que de su uso se desprenden; de esta forma podemos referirnos a:

- su representación visual,

- su capacidad interactiva,

- su simultaneidad,

- su enfoque global,

- su perspectiva relacionante y conectiva,

- su filosofía sistemática, propia de sus lenguajes y por tanto de su funcionamiento.

Todo ello se adscribe perfectamente a las funciones motivantes y estimulantes de la acción cerebral propia del hemisferio cerebral derecho, por lo que la utilización del ordenador se nos presenta como la herramienta más capaz que hoy en día poseemos para su estimulación y desarrollo funcional; con ello se posibilita la conexión entre las aportaciones de la neurociencia y el desarrollo de estilos cognitivos, en este caso de un estilo cognitivo sistémico y propio de la inteligencia creativa e innovadora. Porque resulta, y ésto es tan sangrante que ni los antitecnólogos se lo merecen, que el hemisferio cerebral derecho es el que controla, entre otras capacidades, el desarrollo del talante creativo, de la sensibilidad estética y de las competencias musicales, junto con la emotividad y la afectividad:

"En el vector opuesto se alinean los sujetos que a la hora de asomarse al exterior a la búsqueda de imágenes con que construir su pensamiento dan un mayor protagonismo al hemisferio derecho, el hemisferio de la visión, las melodias, el color, las emociones... etc. Son los denominados 'dependientes de campo', impulsivos, visuales... Dejándose guiar sobre todo por sus referentes externos, adquieren una mayor habilidad en las percepciones de impacto (reconocimiento de rostros y sus emociones y demás configuraciones globales). Configuraciones no sólo figurativas, sino también todas las a ellas asociadas mediante una activa imaginación revisualizadora, como serian los colores... el ritmo... las melodias, armonias...; con mayor habilidad intuitiva, inductiva, inquisitiva, divergente y creativa. Más subjetivos, emotivos y afectivamente más vulnerables, son igualmente menos narcisistas y manifiestan, en general menos preocupación por el éxito y la aprobación social, 18 .

16. Véase sobre el pensamiento sistémico y la posibilidad de una educación en este sentido: Castillejo, J. L. \& Colom, A. J.: Pedagogía Sistémica, Edit. Ceac, Barcelona, 1987.

17. Véase el interesantísimo trabajo de Verle Williams, L.: Aprender con todo el cerebro. Edit. Martínez Roca, Barcelona, 1986.

18. En la misma línea que el ibidem, cabe aquí referir el reciente trabajo de: Doval Salgado, L., Santos Rego, M., Jorge Barreiro, F. J. y Crespo Abelleira, A.: "Estilos docentes y discentes: Consideraciones pedagógicas a la luz de la neurociencia", pp. 311 a 323 de Revista Española de Pedagogía, Año LI, no 195, Madrid, 1993. Vid. p. 315. 
O sea, las dimensiones más significativamente humanísticas. Es por tanto posible hablar de un nuevo estilo de educación, beneficioso para el hombre y la humanidad, que se alumbra mediante la utilización de las denominadas nuevas tecnologías, generadoras del desarrollo de un nuevo conocimiento, fundamentalmente, a través de nuevos entornos educativos.

El mundo virtual del futuro será fruto de las nuevas tecnologías y la educación debe arbitrarse para su recepción. En ella basculará el éxito o el fracaso de la humanidad ante los nuevos retos y posibilidades que la propia humanidad, con el saber, ha ido descubriendo. La nueva concepción de la educación depende pues de la creación de nuevos ambientes educativos, o sea, de ambientes artificiales propiciadores de desarrollo cognitivo virtual.

Parece ser que el futuro ha comenzado ya. Los que vivimos aún —en los límites de lo que nos dejan- en el contexto de cada día más ilusorio de la modernidad, trabajando en el campo de la educación, debemos cumplimentar una obligación moral más, acaso la última; simplemente anunciar y preavisar de la nueva era. Creemos que en parte ya convive aquí, con nosotros. De todas formas, como en los matrimonios, el problema no es de convivencia, sino de supervivencia ${ }^{19}$.

\section{BiBLIOGRAFÍA}

ENZENSBERGER, H. M. (1972): Elementos para una teoria de la comunicación, Madrid, Anagrama.

Colom, A. J. (1985): «Educación y Municipios», pp. 33 a 54 de: CAstillejo, J. L.; Colom, A. J. \& AlTER: Condicionamientos socio-políticos de la educación, Barcelona, Edit. Ceac.

Colom, A. J. (1994): “Tecnología, educación y conocimiento virtual", pp. 17 a 25 de: Tecnología y comunicación educativa, México, $\mathrm{n}^{\mathrm{o}} 22$, enero-marzo.

Colom, A. J. \& Melich, J. C. (1995): Después de la Modernidad, Barcelona, Edit. Paidós.

COLOM, A. J. \& MELICH, J. C. (1995): "Postmodernidad y educación. La teoría de Toffler y la práctica de la $\mathrm{CMU}_{\text {, }}$ en Teoría de la Educación, no 5 , Salamanca.

Castillejo, J. L.; Colom, A. J. \& Alter (1985): Condicionamientos socio-políticos de la educación, Barcelona, Edit. Ceac.

Castillejo, J. L. \& Colom, A. J. (1987): Pedagogía Sistémica, Barcelona, Edit. Ceac.

Danzín, A. (1976): Science et renaissance de l'Europe, Paris, Edit. Chotard.

Doval Salgado, L.; Santos Rego, M.; Jorge Barreiro, F. J. \& Crespo Abelleira, A. (1993): "Estilos docentes y discentes: Consideraciones pedagógicas a la luz de la neurociencia", pp. 311 a 323 de Revista Española de Pedagogía, Madrid, Año LI, no 195.

FinKIEKRAUT (1987): La derrota del pensamiento, Barcelona, Anagrama.

Foster, Habermas, Baudrillard \& otros (1985): La postmodernidad, Barcelona, Edit. Kairós. GINER, S. (1987): Ensayos civiles, Barcelona, Edic. 62.

19. Las ideas aquí expresadas son fruto de la evolución desarrollada a partir de dos textos mucho más amplios que fueron expuestos además bajo otros contextos y orientaciones diferentes en dos ocasiones: Repensar la educación ante el reto tecnológico. Conferencia inaugural del Congreso Informática Educativa, organizado por el Gobierno del Estado de Nuevo León, en Monterrey, México del 17 al 19 de octubre de 1994, y Educació, tecnologia i coneixement virtual, ponencia integrada dentro de las actividades de las II Converses Pedagògiques de la Universitat de Barcelona organizadas por la Fundación Santa María, Barcelona, 11 y 12 de enero de 1996. 
Habermas, J. (1984): Ciencia y técnica como ideología, Madrid, Edit. Técnos.

Habermas, J. (1988): Teoria de la acción comunicativa (2 vols.), Madrid, Edit. Taurus.

HABERMAS, J. (1991): El discurso filosófico de la modernidad, Madrid, Edit. Taurus.

JAMESON (1991): El postmodernismo o la lógica cultural del capitalismo avanzado, Barcelona, Edit. Paidós.

KLAPPENBaCh, A. (1991): Ética y postmodernidad, Madrid, ICE de la Universidad de Alcalá de Henares.

Kung (1991): Proyecto de una ética mundial, Madrid, Edit. Trotta.

Luhmann, N. (1990): «Presupuestos estructurales de una pedagodía reformista. Análisis sociológico de la pedagogía moderna", en Revista de Educación nํ2, Madrid, eneroabril.

Luhmann, N. \& Schorki, K. E. (1993): El sistema educativo. Problemas de reflexión, México, Instituto Tecnológico y de Estudios Superiores de Occidente, Universidad de Guadalajara y Universidad Iberoamericana.

LuHmann, N. (1996): Teoría de la sociedad y pedagogía, Barcelona, Edit. Paidós.

LYOTARD, J. L. (1997): La condición postmoderna, Madrid, Edit. Cátedra.

MCCunTOCK, R. (1993): "Elaboración de un nuevo sistema educativo", pp. 127 a 176 de: McClintock, R.; STREIble, M. \& VÁzQuez, G.: Comunicación, tecnología y diseños de instrucción. La construcción del conocimiento escolar y el uso de los ordenadores, Madrid, CIDE, Ministerio de Educación y Ciencia.

MCClintock, R.; StrReibel, M. \& VÁzquez, G. (1993): Comunicación, tecnología y diseños de instrucción. La construcción del conocimiento escolar y el uso de los ordenadores, Madrid, CIDE, Ministerio de Educación y Ciencia.

Primer Congreso Mundial de Ciudades Educadoras 1990): La ciudad educadora, Barcelona, Ayuntamiento de Barcelona.

SeRVAN-SChreIBer, J. J. \& CRECINE, B. (1986): La revolución del conocimiento, Barcelona, Edit. Plaza y Janés.

SimON, H. (1973): Las ciencias de lo artificial, Barcelona, Edit. ATE.

VATtimo (1986): El fin de la modernidad, Barcelona, Edit. Gedisa.

VAтtrmo (1990): La sociedad transparente, Barcelona, Edit. Paidós.

VÁzQUEZ, G. (1993): "Inteligencia, tecnología y escuela en la sociedad post-industrial", pp. 207 a 252 de MCCLINTOCK, R.; STREIBel, M. \& VÁzQuez, G.: Comunicación, tecnología y diseños de instrucción. La construcción del conocimiento escolar y el uso de los ordenadores, Madrid, CIDE, Ministerio de Educación y Ciencia.

Verle Williams, L. (1986): Aprender con todo el cerebro, Barcelona, Edit. Martínez Roca. 